\section{Adherence and Persistence Among Chronic Myeloid Leukemia Patients During Second-Line Tyrosine Kinase Inhibitor Treatment}

In a study published last year in the October 2014 issue of the Journal of Managed Care E Specialty Pharmacy, Trivedi et al. compared treatment patterns of dasatinib and nilotinib as secondline tyrosine kinase inhibitors (TKI) therapy in chronic myeloid leukemia (CML) patients during the first year of treatment. ${ }^{1}$ Trivedi et al. concluded that among second-line TKI-treated patients, dasatinib patients had significantly higher adherence and lower discontinuation rates compared with those receiving nilotinib. We feel that although the study presents results that are of particular interest to the CML community, certain aspects of the study design raise questions, and the conclusions need to be cautiously considered, since they appear not to be fully substantiated by some of the presented results.

First, the study stated that the recommended nilotinib dose for second-line treatment was $400 \mathrm{mg}(2 \times 200 \mathrm{mg})$ in the Introduction and Subject Selection sections of the article. However, the dose approved by the U.S. Food and Drug Administration for second-line nilotinib is $400 \mathrm{mg}$ twice daily $(2 \times 2$ capsules of $200 \mathrm{mg})$ for a total of $800 \mathrm{mg}$ daily. ${ }^{2}$ A clarification on whether this was a typo or this was the dose used to select nilotinib patients in the study is needed.

Second, by study design the dasatinib cohort was restricted to patients with a dasatinib dose $\leq 100 \mathrm{mg}$. Since dasatinib $140 \mathrm{mg}$ is approved for patients in advanced phase (i.e., accelerated phase or blast crisis), and nilotinib $400 \mathrm{mg}$ twice daily is approved for patients in chronic phase and accelerated phase, using this exclusion criterion on dose for the dasatinib cohort may potentially have led to an imbalance in the proportion of patients in advanced phase between the 2 cohorts. In claims data, as acknowledged by the authors, phase of CML is not identifiable. Therefore, such potential imbalance in the proportion of patients in advanced CML phase between the 2 cohorts is unlikely to be adequately adjusted despite the use of the inverse probability treatment weighting method and the adjustment for the cancer complexity index (developed by Darkow et al. 2007). ${ }^{3}$ However, it is unclear how this potential selection bias may have impacted results, since there is a lack of information on the proportion of second-line TKI use for advanced CML patients in real-world practice and on whether adherence in patients with advanced CML is different from that of patients in chronic phase.

Third, by selecting patients who had at least 2 consecutive prescription claims for the same TKI, the generalizability of the findings of this study may be limited to patients who tolerated their treatments well enough to fill a second and subsequent prescriptions. As acknowledged by the authors in the Discussion section, this may explain to a certain degree the inconsistency in findings from this study and prior studies using similar data that have compared treatment patterns between dasatinib and nilotinib regardless of the number of prescription fills., ${ }^{4,5}$ However, this limitation on the generalizability of their findings should have been reported in the Limitations section.

Finally, the conclusions are not fully supported by all the results reported in the study. For instance, while dasatinib was shown to be associated with a greater adherence based on medi- cation possession ratio (MPR), no statistical difference was found based on proportion of days covered (PDC). MPR and PDC are commonly used to measure treatment adherence. The PDC is considered a more conservative metric for adherence because it corrects for the potential double-counting of days of supply in the MPR calculation, which could lead to overadherent values (i.e., MPR $>1)^{6-8}$ In addition, while Trivedi et al. found the dasatinib cohort to be associated with a lower discontinuation rate based on a 90-day gap, they did not find statistical difference between cohorts based on a 60-day gap nor when adding back patients with only 1 prescription fill of the index second-line TKI.

In summary, because of these concerns regarding the study design and the robustness of the findings, results of the Trivedi et al. study need to be interpreted carefully when making treatment decisions. We look forward to the authors' clarifications of the points raised in this letter.

\section{Lei Chen, MD, PhD}

Director, HEEOR Oncology US CDEMA

Novartis Pharmaceuticals Corporation

lei.chen@novartis.com

\section{Eric Q. $\mathrm{Wu}, \mathrm{PhD}$}

Managing Principal

Analysis Group, Inc.

Eric.Wu@analysisgroup.com

\section{DISCLOSURES}

Chen is an employee of Novartis Pharmaceuticals Corporation. Wu is an employee of Analysis Group, Inc., which has received funding from Novartis Pharmaceuticals Corporation.

\section{REFERENCES}

1. Trivedi D, Landsman-Blumberg P, Darkow T, Smith D, McMorrow D, Mullins CD. Adherence and persistence among chronic myeloid leukemia patients during second-line tyrosine kinase inhibitor treatment. J Manag Care Spec Pharm. 2014;20(10):1006-15. Available at: http://www.amcp.org/ JMCP/2014/October/18585/1033.html

2. Tasigna (nilotinib) capsules. Novartis Pharmaceuticals. Revised October 2007. Available at: http://www.accessdata.fda.gov/drugsatfda_docs/ label/2007/022068lbl.pdf. Accessed September 11, 2015.

3. Darkow T, Henk HJ, Thomas SK, et al. Treatment interruptions and nonadherence with imatinib and associated healthcare costs: a retrospective analysis among managed care patients with chronic myelogenous leukaemia Pharmacoeconomics. 2007;25(6):481-96.

4. Wu EQ, Johnson S, Beaulieu N, et al. Healthcare resource utilization and costs associated with non-adherence to imatinib treatment in chronic myeloid leukemia patients. Curr Med Res Opin. 2010;26(1):61-69.

5. Guérin A, Chen L, Wu EQ, Ponce de Leon D, Griffin JD. A retrospective analysis of therapy adherence in imatinib resistant or intolerant patients with chronic myeloid leukemia receiving nilotinib or dasatinib in a realworld setting. Curr Med Res Opin. 2012;28(7);1155-62.

6. Martin BC, Wiley-Exley EK, Richards S, Domino ME, Carey TS, Sleath BL. Contrasting measures of adherence with simple drug use, medication switching, and therapeutic duplication. Ann Pharmacother. 2009:43(1):36-44.

7. Peterson AM, Nau DP, Cramer JA, Benner J, Gwadry-Sridhar F, Nichol M. A checklist for medication compliance and persistence studies using retrospective databases. Value Health. 2007;10(1):3-12.

8. Cramer JA, Roy A, Burrell A, et al. Medication compliance and persistence: terminology and definitions. Value Health. 2008;11(1):44-47. 


\section{The Authors Respond}

I thank Chen and $\mathrm{Wu}$ for their comments and am grateful for the opportunity to clarify the conclusions noted.

First, the dosing of nilotinib was in error and was missed during both internal and external reviews. The correct dose is $400 \mathrm{mg}$ twice daily for a total of $800 \mathrm{mg}$ daily.

Although patients receiving dasatinib $140 \mathrm{mg}$ were excluded from the study, the unadjusted medication possession ratio (MPR) for this population was $0.82 \pm 0.20$ unweighted and unadjusted. This results in a pooled MPR for dasatinib of $0.86 \pm 0.21$ and a mathematical, but not statistically significant, difference when compared with nilotinib $(P=0.207)$. However, a nonsignificant difference was found for dasatinib $100 \mathrm{mg}$ compared with nilotinib prior to weighting and balancing the populations.

The exclusion-that our findings may be limited to those who tolerated their treatment-from the Limitations section was an oversight. However, since this limitation is addressed in the Discussion section, the reader is adequately notified without it also being in the listed limitations.

Proportion of days covered (PDC) results should be similar to MPR results in general; however, this is not known to be true for this population or for this drug class. The advantage of using MPR is that it accounts for the patient's discontinuation of a drug. ${ }^{1}$ MPR would need to be recalculated using the 60-day gap for discontinuation to see if the difference remains statistically significant. Both of the PDC rates-2 claims weighted and adjusted favoring dasatinib, and 1 claim unweighted and unadjusted favoring neither treatment—contradict the results of Wu et al., who over 180 days found that PDC favored nilo- tinib. ${ }^{2}$ This contradiction demonstrates that additional studies should be performed to see which product on balance has a more favorable adherence profile.

Finally, results of all studies should be interpreted carefully. Our results, in combination with those from studies evaluating similar outcomes, should be evaluated as a whole and combined with additional clinical and patient-specific information when making treatment decisions..$^{2-4}$

\section{Pamela B. Landsman-Blumberg, MPH, DrPH}

Senior Director \& Team Lead Real World Evidence, Xcenda, LLC Pamela.blumberg@xcenda.com

\section{DISCLOSURES}

Landsman-Blumberg was an employee of Truven Health Analytics when the study was conducted and is an employee of Xcenda, LLC, both of which receive funding from Bristol-Myers Squibb.

\section{REFERENCES}

1. Nau DP. Proportion of days covered (PDC) as a preferred method of measuring medication adherence. Available at: http://ep.yimg.com/ty/cdn/epill/ pdcmpr.pdf. Accessed October 14, 2015.

2. Wu EQ, Johnson S, Beaulieu N, et al. Healthcare resource utilization and costs associated with non-adherence to imatinib treatment in chronic myeloid leukemia patients. Curr Med Res Opin. 2010;26(1):61-69.

3. Guérin A, Chen L, Wu E, Ponce de Leon D, Griffin J. A retrospective analysis of therapy adherence in imatinib resistant or intolerant patients with chronic myeloid leukemia receiving nilotinib or dasatinib in a real-world setting. Curr Med Res Opin. 2012;28(7):1155-62.

4. Yood MU, Oliveria SA, Cziraky M, Hirji I, Hamdan M, Davis C. Adherence to treatment with second-line therapies, dasatinib and nilotinib, in patients with chronic myeloid leukemia. Curr Med Res Opin. 2012;28(2):213-19. 